\title{
DEVELOPMENT OF THE COLLECTIVE INTERACTION KLYSTRON (CIK)
}

\author{
J.A. Pasour, T.P. Hughes, and K. Thomason \\ Mission Research Corporation \\ 8560 Cinderbed Rd., Suite 700, Newington, VA 22122
}

\begin{abstract}
The CIK is a microwave source which relies on the negative mass instability for electron bunching, as opposed to the linear-drift ballistic bunching of conventional klystrons. We are testing a proof-of-principle (POP) CIK to verify the predictions of our analyses, which indicate that the $\mathrm{CIK}$ is feasible in the $2-10 \mathrm{GHz}$ region at $\mathrm{MW}$ to $\mathrm{GW}$ power levels. Such a device can be quite compact, and 3 -D numerical simulations show strong particle bunching for efficient operation. The POP device is designed for a $500 \mathrm{keV}, 100 \mathrm{~A}$ electron beam with initial operation at $5 \mathrm{GHz}$. For maximum flexibility and to allow testing over a wide range of parameters, the POP device is modular, incorporating a multisection, helical beam tube to allow variation of the interaction length. Results of the analyses and status of the experiment will be presented.
\end{abstract}

\section{Introduction}

The Collective Interaction Klystron (CIK) utilizes the negative-mass instability to strongly bunch electrons, which are then used to generate microwave radiation. The basic idea of the device is that the negative mass instability can be used to enhance the bunching of an electron bearn which is forced to follow a circular trajectory. The present work was motivated by the theoretical work of Lau and Chernin, ${ }^{1-3}$ who analyzed several general aspects of charged particle circular motion in applied electric and magnetic fields. Lau ${ }^{t}$ originally proposed the CIK and predicted that growth rates of the instability could be large enough to significantly decrease significantly the time and distance required to bunch the beam, as compared to simple ballistic bunching. Also, the growth rate of the instability increases with beam current. Consequently, the CIK could in principle have an important advantage over a conventional klystron, which typically utilizes a number of cavities in a linear array to enhance bunching.

A simple schematic diagram of the CIK as proposed in Ref. 1 is shown in Fig. 1. The basic idea is quite simple. An initial modulation produced at the input cavity excites the negative-mass instability, which produces strong beam bunching in the interaction region. The output cavity is located at the point of optimal bunching to extract energy efficiently from the bunched beam.

We have performed a more complete study to determine the potential of the CIK as an efficient, compact, high-power microwave source. By developing a more complete theory of the device and performing numerical simulations for particular parameters, we have verified many of the attractive features predicted by Lau (high growth rate, strong bunching, and simplicity). Our theoretical analysis includes the effects of an external focusing field, which is required to confine the electrons

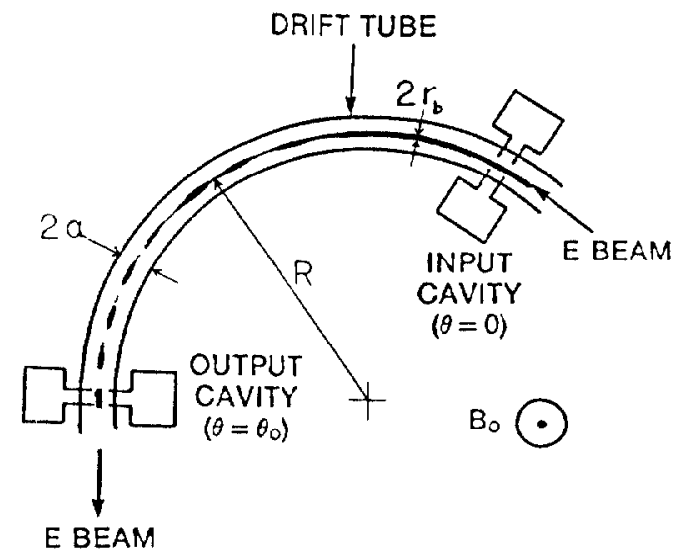

Figure 1: CIK configuration from Lau's paper. ${ }^{1}$ in a tight ring. In contrast, Lau included only the fields necessary for confinement in a long layer. We find that the additional focusing field has a dramatic effect on the interaction, especially at low beam energy, and limits the parameter space over which strong growth of the negative-mass instability can occur.

Our approach employs two MRC-developed codes: a numerical dispersion-relation solver (BTRSQ) and a 3-D, fully relativistic particlein-cell code (IVORY). We have used these codes to determine optimum parameters and to analyze saturation effects, limitations die to beam temperature, different types of beam focusing, and the effects of different types of modulation of the injected electron beam. Based upon our analysis, we have designed and built a proof-of- principle experiment: to demonstrate the attractive features of the CIK in the laboratory. This POP design uses a $100 \Lambda$ electron bcam at an cnergy of 250-500 $\mathrm{keV}$. The device is designed to generate $\sim 10 \mathrm{MW}$ of output at a frequency of $5 \mathrm{GHz}$. A simulation at a beam energy of $1.5 \mathrm{MeV}$ suggests that a multi-GW device is feasible. Such devices have potential applications to high-gradient of accelerators, plasma heating, and various military systems.

\section{Negative Mass Instability}

The negative mass instability has been extensively studied. ${ }^{4-7}$ For a beam of current $I_{b}$, radius $r_{b}$, and energy $\gamma_{0} m c^{2}$ traveling in a circle of radius $R$ in a conducting torus of minor radius $a$, the negative mass dispersion relation can be readily solved to give the following growth rate:

$$
\Gamma_{0}=\frac{\ell c}{R}\left[\frac{\nu\left(1+2 \ln a / r_{b}\right)}{\gamma_{0}^{3}}\left(\frac{1}{\sigma_{r}^{2}}-\frac{1}{\gamma_{0}^{2}}\right)\right]^{1 / 2}
$$

where $\nu=I_{b} / 17 \beta \mathrm{kA}, \ell=\omega / \omega_{0}$ is the azimuthal mode number (operating frequency $\div$ rotation frequency), and $\sigma_{r}$ is the "turie" of the radial focusing. For example, $\sigma_{r}=\sqrt{1-n}$ in a conventional betatron, where $n=-(r / B) d B / d r$ is the betatron field index. Since $0<n<1$ is required for orbital stability, we see that $\sigma_{r}<1$ so that the hetatron is always in the negative-mass regime.

Equation (1) agrees with Lau's growth rate ${ }^{1}$ if we assume $\sigma_{r}-1$ $(n=0)$. For an $n=0$ magnetic field, however, there is no focusing along the $z$-direction, so the injected beam in Fig. 1 would expand along $z$ due to its spacc-charge. The amount of space-charge which can be confined by a focusing system which has radial and vertical tunes $\sigma_{r}=\sigma_{z}=\sigma$ is given by

$$
n_{s} \equiv \frac{2 \nu R^{2}}{\gamma_{0}^{3} \beta_{0}^{2} r_{b}^{2}}<\sigma^{2}
$$

The quantity $n_{*}$ is called the self-field index. In order to achieve the peak current density, the beam must be injected so that it spins poloidally at the local Larmor frequency $e B_{d} / 2 \gamma_{0} m c$. This means that the cathode must be shielded from the magnetic field, so that the beam crosses field lines to enter the drift-tube. Inserting Eq. (2) into Eq. (1), we find that the instability growth rate is bounded by

$$
\Gamma_{0} \leq \frac{\ell v_{\theta 0} r_{b}}{R^{2}}\left[\frac{1}{2}\left(1+2 \ln \frac{a}{r_{b}}\right)\left(1-\frac{\sigma_{r}^{2}}{\gamma_{0}^{2}}\right)\right]^{1 / 2} .
$$

Note that the peak growth-rate is independent of the beam current, due to the fact that the transverse focusing must increase as the current increases. Also, there is no instability for $\gamma_{0}<\sigma_{r}$.

The weak focusing $(\sigma=1 / \sqrt{2}$ ) of the conventional betatron severely restricts the beam current density which can be transported. In order to increase the current density, auxiliary "strong focusing" with $\sigma \gg 1$ is employed. The type of strong focusing we will consider most frequently is that provided by solenoids of alternating polarity placed on the drift-tube. By averaging over several solenoids, one obtains an 
effective radial tune,

$$
\sigma_{r}^{2}=1-n+\frac{1}{4}\left\langle\frac{B_{\theta}^{2}}{B_{z}^{2}}\right\rangle
$$

where $B_{z}$ is the vertical magnetic field. Assuming $n=1 / 2$, we find that the beam is unstable approximately for

$$
\gamma_{0}>\left(\frac{2 \nu R^{2}}{r_{b}^{2}}\right)^{1 / 5}
$$

Focusing using a de toroidal field is also commonly used, as in the modified betatron. ${ }^{8}$ However, the radial and vertical beam motions are coupled in this case, and the expressions we have used up to now involving separate radial and vertical tunes cannot be applied. This problem has been analyzed extensively, ${ }^{9-12}$ and one finds that the beam is unstable approximately for

$$
\gamma_{0}>\left(\frac{4 \nu R^{2}}{a^{2}}\right)^{1 / 3} .
$$

The effect of strong focusing is illustrated in Fig. 2 for the $\ell=30$ mode on a 40 A beam in a drift-tube with a major radius of $25 \mathrm{~cm}$. Alternating-solenoid focusing is assumed. In one case, the beam has a minor radius of $1 \mathrm{~cm}$, and in the other, the focusing is increased to squeeze the beam to a radius of $0.5 \mathrm{~cm}$. In each case, the beam is assumed to be in the Brillouin limit, i.e., $n_{s}=\sigma^{2}$, until $n_{s}$ drops below $1 / 2$, at which point no solenoid focusing is required to maintain an equilibrium. In agreement with Eq. (3), the instability threshold moves up and the peak growth rate decreases for the stronger focusing. The growth rates in Fig. 2 were obtained using the code BTRSQ, which is expected to yield more accurate solutions than Eq. (1) for the high $\ell$-numbers we are most concerned with. In particular, the growth rate increases more slowly with mode number than the linear scaling predicted by Eq. (1). The departure from linear scaling becomes a significant consideration at high $\ell$-numbers because the damping effect of circulation frequency spread scales linearly with $\ell$. The growth rates for alternating and d.c. toroidal fields are generally about the same, but we will show in the simulations below that the nonlinear evolution is quite different.

In order to operate the CIK as an amplifier, one must be able to excite a low-amplitude perturbation which then grows exponentially at the rate given approximately by Eq. (1). In Ref. 1, only the beam voltage is modulated, as in a conventional klystron. To excite a negative-mass mode, however, it is quite beneficial to give the beam a transverse displacement which corresponds to the energy modulation. This modulation positions the electrons at the proper radii for maximum negative-mass interaction; i.e., so that the slower particles immediately begin to overtake the faster ones. The amplitude of the transverse displacement is determined from

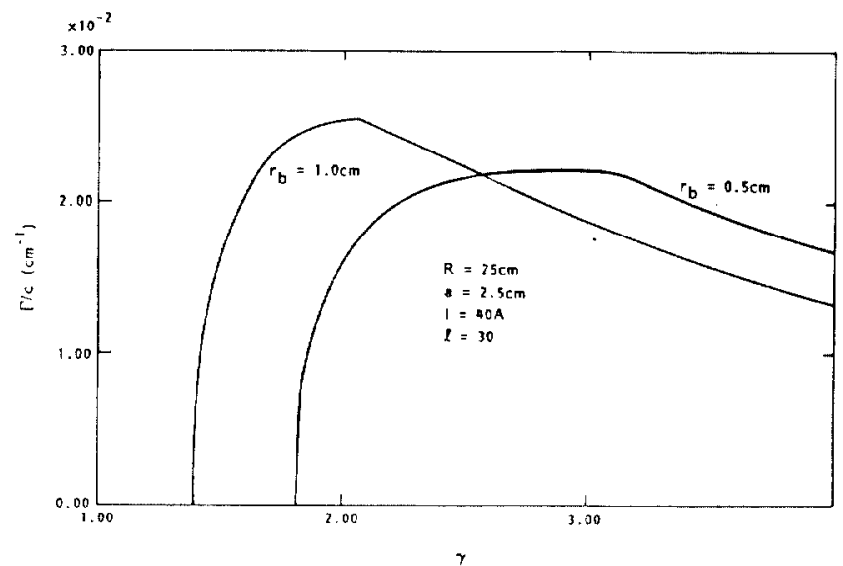

Figure 2: Growth rates of the $\ell=30(5 \mathrm{GHz})$ mode as a function of $\Gamma$ for beam radii of 0.5 and $1.0 \mathrm{~cm}$.

$$
\frac{\delta R}{R}=\frac{\delta \gamma}{\beta_{0}^{2} \gamma_{0} \sigma_{r}^{2}}
$$

Simulation results demonstrating the benefit of optimal excitation of the beam are presented below.

\section{Numerical Simulations of the CIK}

We have performed numerical simulations of several cases which are predicted by BTRSQ to have reasonably large growth rates. The simulations allow us to verify these growth rates as well as to determine the start-up and nonlinear saturation of the instability. The first case we present corresponds to the POP experiment. We assume a $40 \mathrm{~A}$, $500 \mathrm{kV}$ beam in an rms toroidal field of about $300 \mathrm{G}$, which is required to give the beam a radius of $0.5 \mathrm{~cm}$. A simulation of the $\ell=30(5 \mathrm{GHz})$ mode on this beam in a de toroidal field gives a linear growth rate of $9.4 \times 10^{-3} \mathrm{~cm}^{-1}$, versus a BTRSQ-predicted rate of $1.3 \times 10^{-2} \mathrm{~cm}^{-1}$. When an alternating toroidal field is substituted for the dc toroidal field, the observed growth rate is $1.3 \times 10^{-2} \mathrm{~cm}^{-1}$, versus a predicted rate of $1.6 \times 10^{-2} \mathrm{~cm}^{-1}$. The nonlinear development of the instability is quite different from that in the dc toroidal field, however, as shown in Fig. 3. This figure shows the electron distribution in the $r-z$ and $r-\theta$ configuration space. In the dc toroidal field, the beam spreads along the $z$ direction duc to $F_{r} \times B_{\theta}$ forces as it tries to move radially. Motion along the $z$ direction merely disrupts the beam without contributing to the toroidal bunching. For the alternating field, this drift is eliminated, and the beam is much better behaved. This observation is one of the principal reasons for our choice of alternating solenoids in the POP experiment.

As discussed above, proper excitation of a negative-mass mode requires both a voltage modulation and a transverse displacement. In Fig. 4, we compare the growth of the rf field energy for a beam which was excited with only a transverse displacement and a beam which was excited with both a transverse displacement and a voltage modulation satisfying Eq. (7). In the first case, the beam drifts for over a meter before clear exponential growth commences. In the second case, growth commences almost immediately. The applied voltage modulation is $\pm 4.4 \mathrm{kV}$, with a corresponding transverse displacement of $\pm 0.07 \mathrm{~cm}$. Peak bunching of the beam is reached in 1.4 major revolutions. This distance could be reduced by increasing the initial perturbation.

It is interesting to note the dramatic difference in bunching between the CIK result and that which would be expected in a simple twocavity linear klystron. In the linear klystron, the bunching ceases to increase at a distance of $\lambda_{p} / 4$ from the bunching cavity, where $\omega_{p}=2 \pi c / \lambda_{p}$ is the plasma frequency. For the beam parameters used
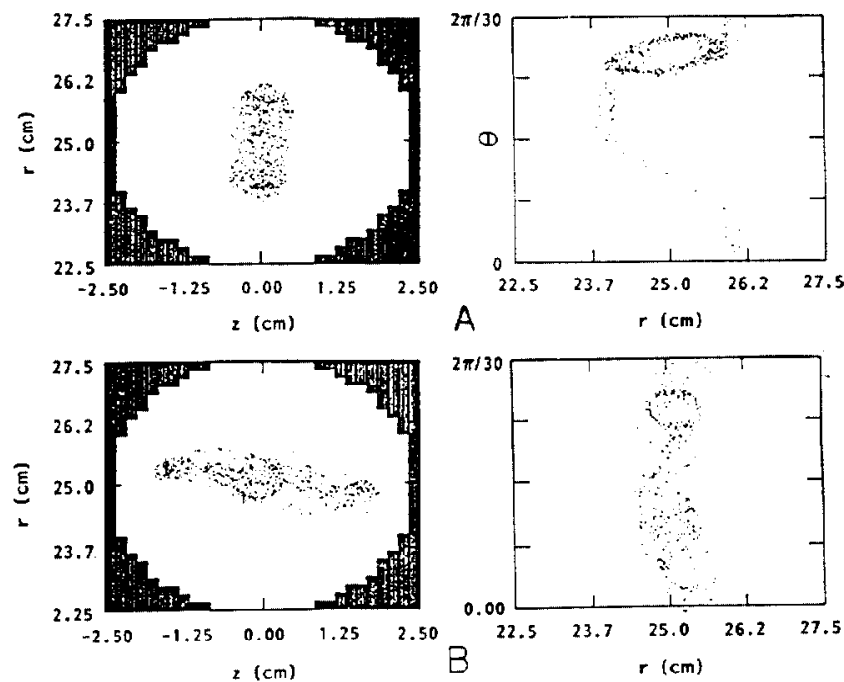

Figure 3: Comparison of nonlinear state of a $40 \mathrm{~A}, 500 \mathrm{kV}$ beam in the presence of (a) alternating and (b) dc toroidal field focusing. 

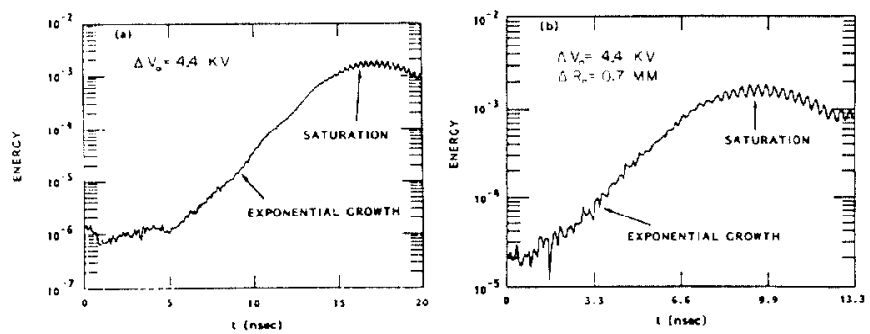

Figure 4: Growth of field energy in the $\ell=30$ ( 5 GHz) mode as a function of time for a beam initialized with (a) a transverse perturbation only, and (b) a transverse perturbation and voltage modulation.

in the simulation, maximum bunching would occur in a distance of only $12 \mathrm{~cm}$. At that point, the space-charge forces would cause the bunch to expand. Given the small modulation amplitude of the simulation, only very weak bunching would occur in this distance. In the CIK, on the other hand, the beam continues to bunch due to the negative-mass irustability.

The power of the beams in the simulations presented thus far has been modest ( $20 \mathrm{MW}$ ). To demonstrate the CIK's high-power capabilities, we performed some simulations at $1.5 \mathrm{MeV}$ and $600 \mathrm{~A}$, keeping the geometry the same but increasing the focusing fields. The growth rate at $3 \mathrm{GHz}$ at the higher power level is similar to that shown in Fig. 4 $(1.2-1.4 \% / \mathrm{cm})$. We also performed a high-power simulation at $10 \mathrm{GHz}$ $(\ell=54)$. Configuration space plots of this simulation are shown in Fig. 5. The growth rate is even higher than at lower frequency, and saturation occurs in less than one turn.

\section{Proof-of-Principle Experiment}

The POP experiment was designed based on the simulations just presented. A beam energy of $400-500 \mathrm{keV}$ and current of $40-100 \mathrm{~A}$ were chosen to give a reasonable growth rate while allowing the use of a relatively simple modulator. The beam tube is a $1.5-\mathrm{cm}-\mathrm{dia}$. stainless steel tube which was bent into a $50-\mathrm{cm}$-dia. helix having a $10 \mathrm{~cm}$ pitch. A helical beam tube was chosen to allow a multi-turn interaction, although an optimized design should allow saturation to occur in less than 1 turn. The beam tube is assembled in sections, so the length can be varied from 1/4 turn to 3 turns. Standard klystronlike $\mathrm{rf}$ cavities are used to excite and couple of energy from the beam. Initial operation uses $5 \mathrm{GHz}$ cavities. The vertical field $(118 \mathrm{G}$ for $500 \mathrm{keV}$ ) is generated by 4 single-turn windings parallel to the beam tube. The alternating toroidal field (up to $500 \mathrm{G}$ ) is provided by a set of $9.5-\mathrm{cm}-\mathrm{dia}$. coils located every $15^{\circ}$ around the tube. This coil configuration is sufficiently compact that the vertical field can be limited to the curved portion of the beam tube and there is minimal field contamination between adjacent turns of the tube. The complete coil assembly is mounted on the helical beam tube using phenolic spacers fabricated in $15^{\circ}$ segments which clamp around the tube. A schematic diagran of the POP device is shown in Fig. 6. All components have been fabricated and tested, and experiments will begin upon the delivery of the modulator.
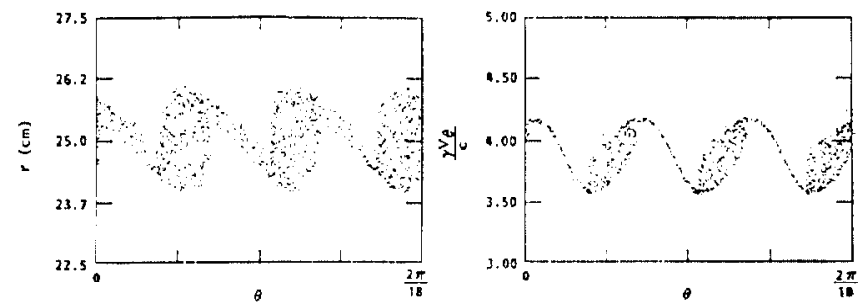

Figure 5: Plots of longitudinal particle positions (left) and momentum (right) at peak bunching for the high-power 10 GHz mode.

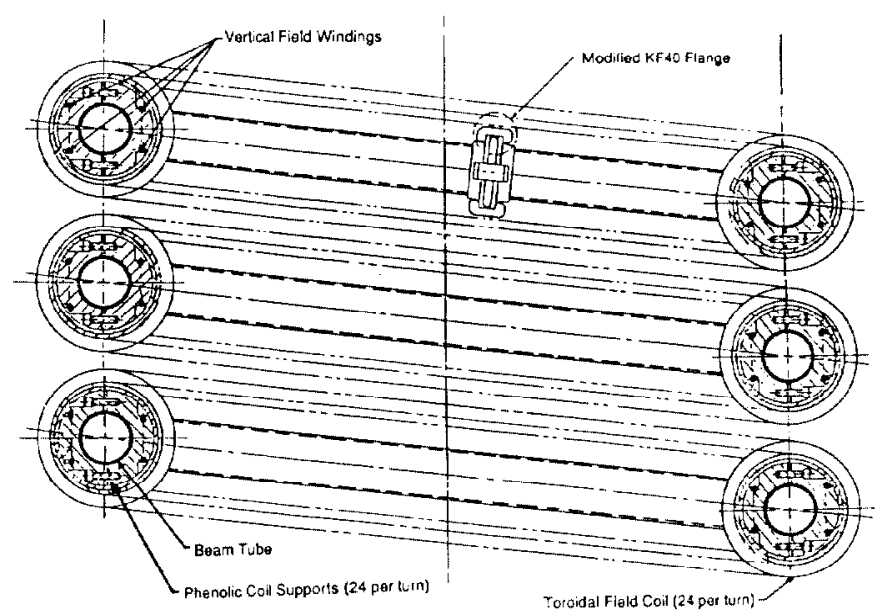

Figure 6: Side view of the multi-turn experimental configuration.

The electron gun for the CIK uses a cold field-emission cathode, which greatly reduced fabrication costs and vacuum requirements. Electrons are emitted from a velvet surface. The gun was designed using the SLAC gun code to produce a $1-\mathrm{cm}$-dia. beam. The gun is powered by a conventional modulator having a $1 \mu$ sec output pulse.

The microwave cavities are of a standard re-entrant design. At the MW power levels of the experiment, power is extracted from the output cavity through a vacuum window and waveguide. The injector geometry can accommodate a second cavity which could provide a transverse modulation, which the simulations have shown to decrease the start-up time. The deflection cavity can be phase locked to the input cavity by driving both from the same source. Fine tuning of the relative phase between the two cavities can be controlled by an external phase shifter.

\section{Conclusions}

The CIK is predicted to be a viable source of high-power microwave radiation. Scaling to frequencies as high as $10 \mathrm{GHz}$ seems favorable, but technical issues such as cavity coupling must be addressed. This same mechanism might also be used with different forms of coupling at higher frequencies. The device is compact and relatively simple A POP experiment designed to verify the theoretical predictions will soon be operational.

This work is supported by the U.S. Department of Energy under Contract \#DE-AC05-87ER80522.

\section{References}

1. Y. Y. Lau, Phys. Rev. Lett. 53, 395 (1984)

2. D. Chernin and Y. Y. Lau, Phys. Fluids 27, 2319 (1984).

3. Y. Y. Lau and D. Chernin, Phys. Rev. Lett. 52, 1425 (1984)

4. C. E. Nielson, A. M. Sessler, and K. R. Symon, Proc. Int'l. Conf. on Accelerators (CERN, Gencva, 1959), p. 239.

5. R. J. Briggs and V. K. Neil, J. Nucl. Energy C9, 209 (1967); Plasma Physics 8, 255 (1966); Plasma Physics 9, 209 (1967).

6. R. W. Landau and V. K. Neil, Phys. Fluids 9, 2412 (1966).

7. V. K. Neil and A. M. Sessler, Rev. Sci. Instrum. 36, 429 (1965)

8. C. A. Kapetanakos, et al., Phys. Fluids, 261634 (1983).

9. B. B. Godfrey and T. P. Hughes, Phys. Fluids 28, 669 (1985).

10. B. B. Godfrey and T. P. Hughes, IEEE Trans. Nucl. Sci. NS32, 2495 (1985).

11. T. P. Hughes and B. B. Godfrey, Appl. Phys. Lett. 46, 473 (1985)

12. T. P. Hughes and B. B. Godfrey, Phys. Fluids 29, 1698 (1986). 